\title{
Towards One Health: Increasing Awareness of Animal Health Among Public Health Stakeholders
}

\author{
Fernanda C. Dórea*1, Céline Dupuy² and Judy E. Akkina ${ }^{3}$ \\ ${ }^{1}$ Swedish Zoonoses Centre, National Veterinary Institute, Uppsala, Sweden; ${ }^{2}$ French Agency for Food, Environmental and \\ Occupational Health and Safety (Anses), Lyon Laboratory, Epidemiology Unit, Lyon, France; '3nited States Department of \\ Agriculture, Animal and Plant Health Inspection Agency, Veterinary Services, Center for Epidemiology and Animal Health, Fort \\ Collins, CO, USA
}

\section{Objective}

To discuss opportunities to improve the synergy between animal and public health and increase awareness, among public health workers, of the concept of animal health.

\section{Introduction}

The recent focus on the "One Medicine" concept has resulted in an increased awareness that the control of diseases in animal populations, whether zoonotic or not, can be of great public health importance. Zoonotic and foodborne diseases represent an immediate threat to the health of human populations, while rapid spreading diseases in animals can compromise the food-supply and the economy of a country or region. On the other hand, animal populations can serve as sentinels, and continued surveillance can prevent the emergence and/ or rapid spread of pathogens potentially harmful to humans. However, awareness of the activities developed in the field of animal health is still low among public health workers. To date, the cooperation between public health and animal health epidemiologists has mainly involved the control of outbreaks of foodborne diseases. Greater cooperation between the two fields, however, could improve prevention and reduce the number of such outbreaks.

\section{Audience Engagement}

The moderators will encourage the discussion first by surveying the awareness of animal health among the ISDS conference participants. Do all public health workers know what is animal health and what it involves? Do they know who is responsible for animal health in their country?

The moderators will then center the discussion on the current situation regarding public and animal health collaborations: Is there already any synergy between human and animal health sector? If yes, what are some examples? The results of a survey through the Triple S project (syndromic surveillance systems in Europe), in which member states were asked similar questions, will be used to guide this discussion and establish comparisons with the situation in other countries. This will open discussion on further improvement for collaboration between both sides: What methods are already shared or could be shared between public and animal health? Why is it important for public health workers to be aware of animal health?

Lastly, the intention is to steer towards a productive discussion of how awareness and synergy can be improved between the two fields. How can public health epidemiologists contribute to animal health? What can we learn from each other? How can we promote this cooperation? What are the current challenges and how can we start to address them?

The facilitators feel strongly that the discussion should lead to a productive outcome, and therefore the discussion will be documented for circulation among public health and animal health stakeholders that are interested in this topic, and the suggestions will be followed up with further debates at future conferences, ISDS webinars and possibly a publication reviewing the issue.

\section{Keywords}

animal health; public health; one health

*Fernanda C. Dórea

E-mail: fernanda.dorea@sva.se 